\title{
Lymphocyte proliferation to mycobacterial antigens is detectable across a spectrum of HIV-associated tuberculosis Timothy Lahey*1, Mecky Matee ${ }^{2}$, Lillian Mtei², Muhammad Bakari², Kisali Pallangyo ${ }^{2}$ and C Fordham von Reyn ${ }^{1}$
}

Address: ${ }^{1}$ Dartmouth Medical School, Lebanon, NH, USA and ${ }^{2}$ Muhimbili University of Health and Allied Sciences, Dar es Salaam, Tanzania Email: Timothy Lahey* - Timothy.Lahey@Dartmouth.edu; Mecky Matee - mmatee@muhas.ac.tz; Lillian Mtei - Indefomiro@yahoo.com; Muhammad Bakari - mbakari@muhas.ac.tz; Kisali Pallangyo - kpallangyo@muhas.ac.tz; C Fordham von Reyn - C.Fordham.von.Reyn@Dartmouth.edu

* Corresponding author

Published: 23 February 2009

BMC Infectious Diseases 2009, 9:21 doi:10.1186/1471-2334-9-2I
Received: 15 September 2008

Accepted: 23 February 2009

This article is available from: http://www.biomedcentral.com/I47/-2334/9/2 I

(c) 2009 Lahey et al; licensee BioMed Central Ltd.

This is an Open Access article distributed under the terms of the Creative Commons Attribution License (http://creativecommons.org/licenses/by/2.0), which permits unrestricted use, distribution, and reproduction in any medium, provided the original work is properly cited.

\begin{abstract}
Background: Identifying novel TB diagnostics is a major public health priority. We explored the diagnostic characteristics of antimycobacterial lymphocyte proliferation assays (LPA) in HIVinfected subjects with latent or active TB.

Methods: HIV-infected subjects with bacille Calmette Guérin (BCG) scars and CD4 counts $\geq 200$ cells $/ \mathrm{mm}^{3}$ entering a TB booster vaccine trial in Tanzania had baseline in vivo and in vitro immune tests performed: tuberculin skin tests (TST), LPA and five day assays of interferon gamma (IFN- $\gamma$ ) release. Assay antigens were early secreted antigenic target 6 (ESAT-6), antigen 85 (Ag85), and Mycobacterium tuberculosis whole cell lysate (WCL). Subjects were screened for active TB at enrollment by history, exam, sputum smear and culture. We compared antimycobacterial immune responses between subjects with and without latent or active TB at enrollment.

Results: Among 1885 subjects screened, 635 had latent TB and 13 had active TB. Subjects with latent TB were more likely than subjects without TB to have LPA responses to ESAT-6 (I3.2\% vs. $5.5 \%, P<0.000 I)$, Ag85 (I8.7\% vs. $3.1 \%, P<0.000 I)$, and $W C L(45.7 \%$ vs. $17.1 \%, P<0.000 I)$. Subjects with active TB also were more likely than those without active TB to have detectable LPA responses to ESAT-6 (38.5\% vs. $8.1 \%, P=0.0001)$, Ag85 (46.2\% vs. $8.5 \%, P<0.0001)$, and WCL $(61.5 \%$ vs. $27.0 \%, P=0.0053)$. In subjects with a positive TST, LPA responses to ESAT-6, Ag85 and $W C L$ were more common during active TB ( $P<0.000$ I for all tests). In diagnosing active TB, in vivo and in vitro tests of mycobacterial immune responses had sensitivity and specificity as follows: TST $84.6 \%$ and $65.5 \%$, ESAT-6 LPA $38.5 \%$ and $92.0 \%$, Ag85 LPA $46.2 \%$ and $91.5 \%$, and WCL LPA $61.5 \%$ and $73.0 \%$. Detectable LPA responses were more common in patients with higher CD4 counts, and higher HIV viral loads.
\end{abstract}

Conclusion: Lymphoproliferative responses to mycobacteria are detectable during HIVassociated active TB, and are less sensitive but more specific than TST.

Trial registration: ClinicalTrials.gov Identifier NCT00052195. 


\section{Background}

Tuberculosis (TB) is a major cause of death in HIVinfected people worldwide [1], yet, current approaches to TB diagnosis in HIV-infected people are inadequate [2]. Therefore, identifying novel TB diagnostics is a major international public health priority [3]. Interferon gamma (IFN- $\gamma$ ) release assays (IGRA) are under active investigation for the diagnosis of latent and active TB [4-7]. Since diminished IFN- $\gamma$ release is a cardinal antimycobacterial immune defect in HIV-infected people [8-14], and vulnerability to active TB in HIV-infected people increases with increasing immunodeficiency [15], HIV-related immunodeficiency has the potential to compromise IGRA accuracy. Thus, it will be important to investigate additional methods of diagnosing latent and active TB.

Antimycobacterial lymphocyte proliferation is also detectable in HIV-infected subjects with latent and active TB [8,16-18], and therefore merits investigation for the diagnosis of latent and active TB in HIV infection. However, because antimycobacterial lymphocyte proliferation can also be impaired during HIV infection $[8,16,17]$, it will be important to evaluate the relation between stage of HIV disease and LPA diagnostic utility. Accordingly, we evaluated LPA responses in subjects with and without latent or active TB in a cohort of HIV-infected BCG-immunized subjects with CD 4 counts $\geq 200$ cells $/ \mathrm{mm}^{3}$ entering a TB booster vaccine trial in Tanzania $[19,20]$. This is the first report to characterize the diagnostic characteristics of LPA during active TB.

\section{Methods}

\section{Study subjects and data collection}

The Dar Dar Study was a phase III randomized placebocontrolled trial of a prime-boost vaccine strategy for the prevention of HIV-associated tuberculosis in adults in Dar es Salaam, Tanzania [20]. Enrollment for this study occurred from 2001 to 2005, and study follow up continued through January 2008. All subjects were screened with a standardized interview, including history of prior treatment for active tuberculosis, physical examination, CD4 count and single view chest $\mathrm{x}$-ray. Eligible subjects who gave informed consent were enrolled if they have two positive ELISA antibody tests for HIV, a CD4 count $\geq 200$ / $\mathrm{mm}^{3}$, a BCG scar and no evidence of active tuberculosis (TB). At enrollment, subjects were further evaluated with three expectorated sputum samples collected for AFB smear and culture, and a single mycobacterial blood culture. Per protocol, subjects with active TB detected immediately at baseline were ineligible for enrollment and thus discharged from the study with immunological studies not performed. In contrast, subjects with baseline active TB that was not detected immediately, but later identified via baseline mycobacterial cultures of sputum or blood, were not yet discharged from the study and as a result had immunological tests performed. Subjects in this latter category, with delayed recognition of baseline TB on whom immunological studies were performed, are the subject of this report. Human experimentation guidelines of the United States Department of Health and Human Services, as well as that of the Committee for the Protection of Human Subjects at Dartmouth College and the Research Ethics Committee of the Muhimbili University of Health and Allied Sciences, were followed in the conduct of this research. This study is registered through the National Institutes of Health (NCT00052195).

\section{Definitions of TB}

Subjects with a reactive tuberculin skin test (TST) at $\geq 5$ $\mathrm{mm}$ but no active TB were deemed to have latent TB. Subjects were designated as having active $\mathrm{TB}$ if they met a study definition of definite or probable TB as determined by the consensus of a three person expert panel (Table 1).

\section{Tuberculin skin testing}

All subjects underwent TST with $0.1 \mathrm{~mL}$ tuberculin (RT23, State Serum Institute, Copenhagen) as well as with 0.1 $\mathrm{mL}$ Mycobacterium avium sensitin (MAS, State Serum Institute, Copenhagen). The size of skin induration at the site at $48-72$ hours was measured, and reactions $\geq 5 \mathrm{~mm}$ were considered positive.

\section{Lymphocyte proliferation assays (LPA)}

LPA were conducted on freshly isolated and ficolled peripheral blood mononuclear cells using a standard five day ${ }^{3} \mathrm{H}$-thymidine incorporation method with media alone, $1 \mathrm{mcg} / \mathrm{ml} \mathrm{M}$. tuberculosis ESAT- $6,0.5 \mathrm{mcg} / \mathrm{ml} \mathrm{M}$. tuberculosis Ag85, or $0.5 \mathrm{mcg} / \mathrm{ml} \mathrm{M}$. tuberculosis whole cell lysate (WCL), with all antigens acquired through NIH, NIAID Contract No. HHSN266200400091C, entitled "Tuberculosis Vaccine Testing and Research Materials," awarded to Colorado State University. After incubation, $20 \mu \mathrm{l}$ of $50 \mu \mathrm{Ci} / \mathrm{ml}^{3} \mathrm{H}$-thymidine was added to wells for 24 hours, after which the cells were harvested onto filter paper and sent to the National Public Health Institute in Helsinki, Finland, for data acquisition on a scintillation counter. Results were expressed as a proliferation index (PI; counts per minute [CPM] of antigen stimulated cells divided by CPM of unstimulated cells), with a proliferation index of $\geq 5$ considered positive. LPA results are shown for all assays, but equivalent results were derived when identical analyses were undertaken after excluding assays if the positive control (phytohemagglutinin [PHA] $2.5 \mu \mathrm{g} / \mathrm{ml}$ ) PI was $<3$, or less than the unstimulated condition, or if the CPM for the unstimulated cells was over 5000 .

\section{Supernatant IFN- levels}

IFN- $\gamma$ levels in frozen cell culture supernatants were evaluated after 5-day stimulation with the same antigens 
Table I: Case definitions.

\begin{tabular}{|c|c|}
\hline Definite TB & $\begin{array}{l}\text { I. One or more sputum cultures positive for Mycobacterium tuberculosis (MTB) with } \geq 10 \text { colony forming units (CFU); or, } \\
\text { 2. Two or more sputum cultures with I-9 CFU of MTB (indeterminate MTB culture); or, } \\
\text { 3. Two or more positive sputum smears for acid fast bacilli (AFB); or, } \\
\text { 4. One or more cultures for MTB from the blood or other sterile body site. }\end{array}$ \\
\hline Probable TB & $\begin{array}{l}\text { I. Positive chest x-ray plus either } \\
\text { a. one positive sputum AFB smear, or, } \\
\text { b. one indeterminate MTB culture result; or, } \\
\text { 2. Clinical symptoms/signs plus either } \\
\text { a. one positive sputum AFB smear, or, } \\
\text { b. one indeterminate MTB culture result; or, } \\
\text { 3. Clinical symptoms/signs and a positive } x \text {-ray plus a response to anti-TB therapy; or, } \\
\text { 4. One positive sputum AFB smear from a sterile site plus clinical symptoms/signs of tuberculosis; or, } \\
\text { 5. Caseous necrosis on a tissue biopsy. }\end{array}$ \\
\hline Latent TB & $\begin{array}{l}\text { I. Tuberculin skin test } \geq 5 \mathrm{~mm} \\
\text { 2. No definite or probable TB. }\end{array}$ \\
\hline AFB, acid fast & acillus; CFU, colony forming units; MTB, Mycobacterium tuberculosis; TB, tuberculosis \\
\hline $\begin{array}{l}\text { using a sta } \\
\text { (ELISA; R\&I } \\
\text { IFN- } \gamma \text { level } \\
\text { ations abov } \\
\text { Statistical a } \\
\text { Standard de } \\
\text { acterize ba } \\
\text { study popu }\end{array}$ & 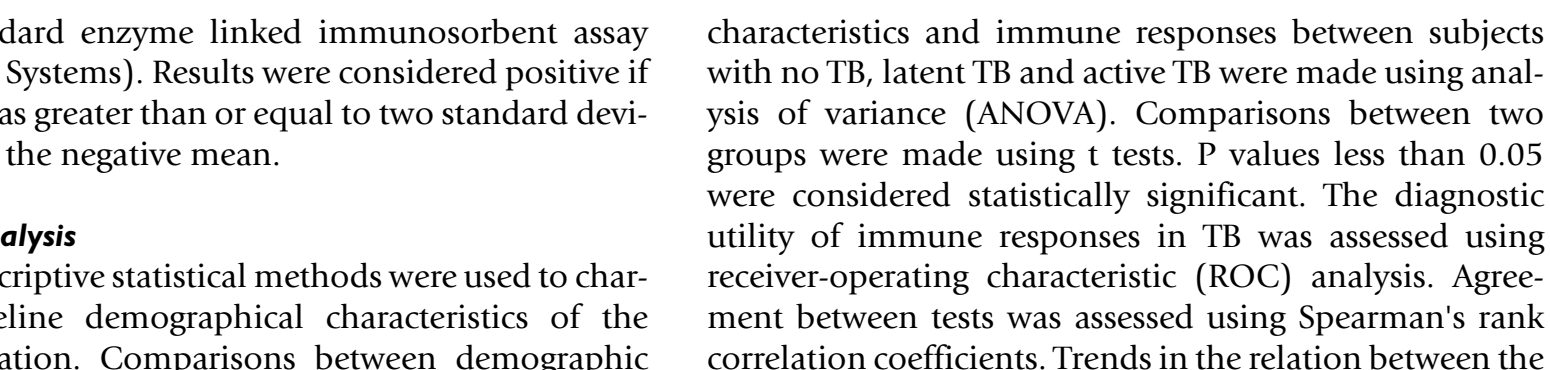 \\
\hline
\end{tabular}

Table 2: Characteristics of study subjects.

\begin{tabular}{|c|c|c|c|c|}
\hline & $\begin{array}{l}\text { Subjects without TB } \\
\qquad(\mathrm{n}=1206)\end{array}$ & $\begin{array}{l}\text { Subjects with latent TB } \\
\qquad(\mathrm{n}=635)\end{array}$ & $\begin{array}{l}\text { Subjects with active TB } \\
\qquad(\mathrm{n}=13)\end{array}$ & P-value by ANOVA \\
\hline Age, mean & 33.0 & 33.5 & 34.1 & 0.5102 \\
\hline Male, \% (n) & 247 (20.5\%) & $193(30.4 \%)$ & $3(23.1 \%)$ & $<0.0001$ \\
\hline CD4 cells $/ \mathrm{mm}^{3}$, mean & 455 & 502 & 376 & 0.0001 \\
\hline HIV viral load, copies $/ \mathrm{mL}$, mean & 37,286 & 28,365 & 87,293 & 0.0063 \\
\hline Previous TB, \% (n) & $98(8.1 \%)$ & $61(9.6 \%)$ & $2(15.4 \%)$ & 0.3865 \\
\hline On antiretrovirals, \% (n) & $44(3.6 \%)$ & $13(2.0 \%)$ & $0(0 \%)$ & 0.1366 \\
\hline TST, mm, mean & 0.2 & 15.5 & 15.0 & $<0.0001$ \\
\hline TST $\geq 5$ mm, \% (n) & $0(0 \%)$ & $635(100 \%)$ & II (84.6\%) & $<0.0001$ \\
\hline MAS, mm, mean & 0.3 & 9.6 & 10.1 & $<0.0001$ \\
\hline MAS $\geq 5$ mm, \% (n) & $835(69.2 \%)$ & $582(91.7 \%)$ & $10(76.9 \%)$ & $<0.0001$ \\
\hline
\end{tabular}

ANOVA = analysis of variance; HIV = human immunodeficiency virus; MAS = Mycobacterium avium sensitin skin test; TB = tuberculosis; TST = tuberculin skin test 
risk of TB and the number of antimycobacterial immune responses were evaluated using the score test for trend of odds. Parameters demonstrating differences between TB and no TB groups in univariate analyses were incorporated into a logistic regression model. Correlations between the results of in vivo and in vitro immune assays, and with clinical parameters, utilized the Pearson chisquared test. Data were analyzed on STATA 9 (College Station, TX) and Graph Pad Prism 4 (San Diego, CA).

\section{Results}

The characteristics of the study subjects are shown in Table 2, including the 13 with delayed recognition of active TB at baseline. All subjects were HIV positive, had BCG scars and CD4 counts $\geq 200 / \mathrm{mm}^{3}$. Evaluable LPA results were available in 1855 subjects: IFN- $\gamma$ results in 1542 subjects and TST results in 1815 subjects. Thirteen subjects had positive sputum cultures for MTB; eleven fit study criteria for definite TB and two for probable TB. Subjects with active TB had higher mean HIV viral loads $(87,292.6$ vs. 34023.0 copies $/ \mathrm{mL}, \mathrm{P}=0.0066)$, and were more likely to have a positive TST $(84.6 \%$ vs. $34.5 \%, \mathrm{P}=$ 0.0002 ) when compared to all subjects without active TB.

\section{LPA results during latent and active TB}

Figure 1A and Table 3 show the LPA responses to mycobacterial antigens in subjects with no TB, latent TB and with active TB. Compared to subjects without TB, detectable LPA responses to ESAT-6, Ag85 and WCL were significantly more common in subjects with latent and active TB. A pattern of increasing LPA responses to all three antigens was seen when moving from no TB to latent $\mathrm{TB}$ to active TB.

\section{Agreement of LPA results}

There was a significant correlation between LPA responses to different mycobacterial antigens: ESAT-6 vs. Ag85, Spearman's rho 0.4242, P $<0.0001$; ESAT-6 vs. WCL, Spearman's rho 0.3646, P < 0.0001; Ag85 vs. WCL, Spearman's rho $0.4033, \mathrm{P}<0.0001$.

\section{LPA responses by TST status during active TB}

We compared LPA responses in subjects with active TB to LPA responses in all other subjects. In subjects with a positive TST, TB was more likely in subjects with detectable LPA responses to ESAT-6 ( $\mathrm{P}<0.0001)$, Ag85 ( $\mathrm{P}<0.0001)$ and WCL $(\mathrm{P}<0.0001)$. The specificity of LPA to ESAT- 6 and Ag85 was over $80 \%$ in subjects with a positive TST (Table 4).

\section{LPA results according to TST status in subjects without active TB}

In the group of patients without active $\mathrm{TB}$ at baseline, detectable LPA responses were more common in subjects with a positive TST: ESAT-6 $13.2 \%$ vs. 5.5\%, $\mathrm{P}<0.0001$;
Ag85 18.7\% vs. 3.1\%, P < 0.0001; and, WCL 45.7\% vs. $17.1 \%, \mathrm{P}<0.0001$.

\section{LPA operating characteristics}

The sensitivity and specificity of TST for detecting TB were $84.6 \%$ and $65.5 \%$, respectively. As shown in Table 4 the sensitivity of LPA assays was lower than TST in every case, but antimycobacterial LPA assays were generally more specific. The area under the ROC curve (AUROC) for TST was higher than for LPA at either PI cutoff. The positive predictive values for LPA responses to all antigens were low, as for TST. The negative predictive values for all LPA assays were approximately 99\% regardless of TST status. Assay operating characteristics were not improved when combining multiple antigen responses (data not shown).

\section{LPA responses and CD4 count or HIV viral load}

There was a weak but statistically significant negative correlation between CD4 count and the likelihood of LPA responses to ESAT-6 (Pearson $\mathrm{r}-0.1044, \mathrm{P}<0.0001$ ), Ag85 (Pearson $\mathrm{r}-0.0744, \mathrm{P}=0.0013$ ) and WCL (Pearson r $-0.1162, \mathrm{P}<0.0001)$. In subjects stratified by CD 4 count, LPA responses showed the same increasing pattern from no TB to latent TB to active TB, but in the low CD4 count group LPA was generally less able to discriminate between latent and active TB (Figures 1B-1C). No LPA response could discriminate subjects with latent TB from active TB in the CD4 < 350 stratum. The HIV viral load correlated weakly but positively with the likelihood of detectable LPA responses to ESAT-6 (Pearson r 0.1577, P < 0.0001), Ag85 (Pearson r 0.1277, P = 0.0008), and WCL (Pearson $r$ 0.1131, $P=0.0029)$. Because of the relation of LPA responses to CD4 count and HIV viral load, we stratified our analysis of the correlation between active TB and the antimycobacterial immune response by CD4 count and, separately, HIV viral load (Table 5), finding that detectable mycobacterial LPA responses were associated with higher odds of TB at higher CD4 counts and higher HIV viral loads.

\section{Multiple LPA responses related to greater TB risk}

Figure 2 shows the relation between the number of antimycobacterial responses by LPA and the odds of TB. An increasing number of antimycobacterial LPA responses were associated with increasing odds of TB $(P=0.0097)$.

\section{Multivariate model}

In a multivariate logistic regression analysis adjusting for factors that differed between subjects with and without active TB, active TB was associated with LPA responses to ESAT-6 (OR 3.8, $\mathrm{P}=0.036$ ) and Ag85 (OR 3.2, $\mathrm{P}=$ $0.0760)$.

\section{IFN- results during latent and active TB}

IFN- $\gamma$ responses were more common in subjects with latent TB compared to those without TB (ESAT-6, 49.3\% 
(A)

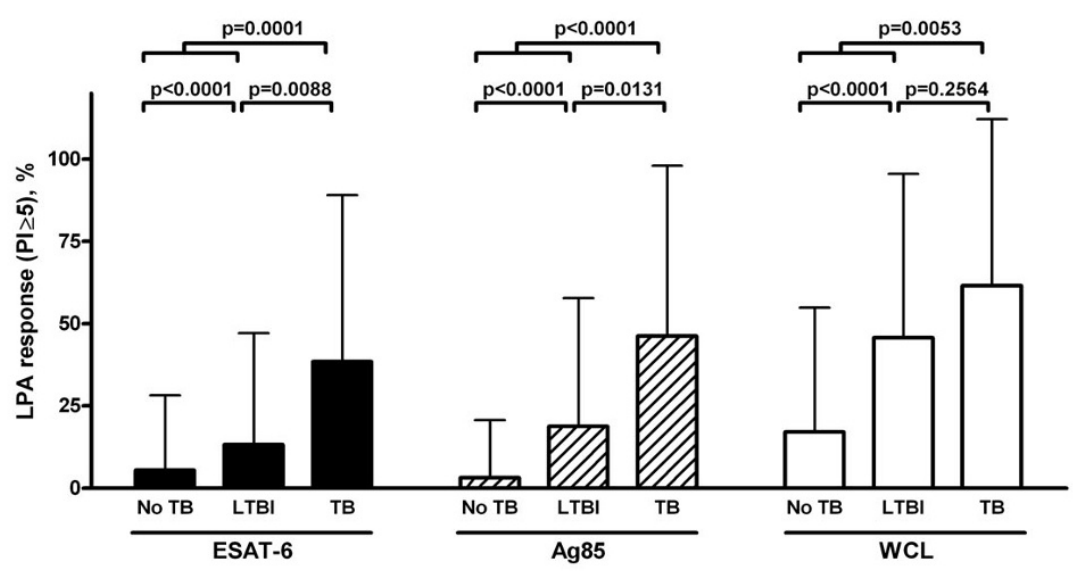

(B)

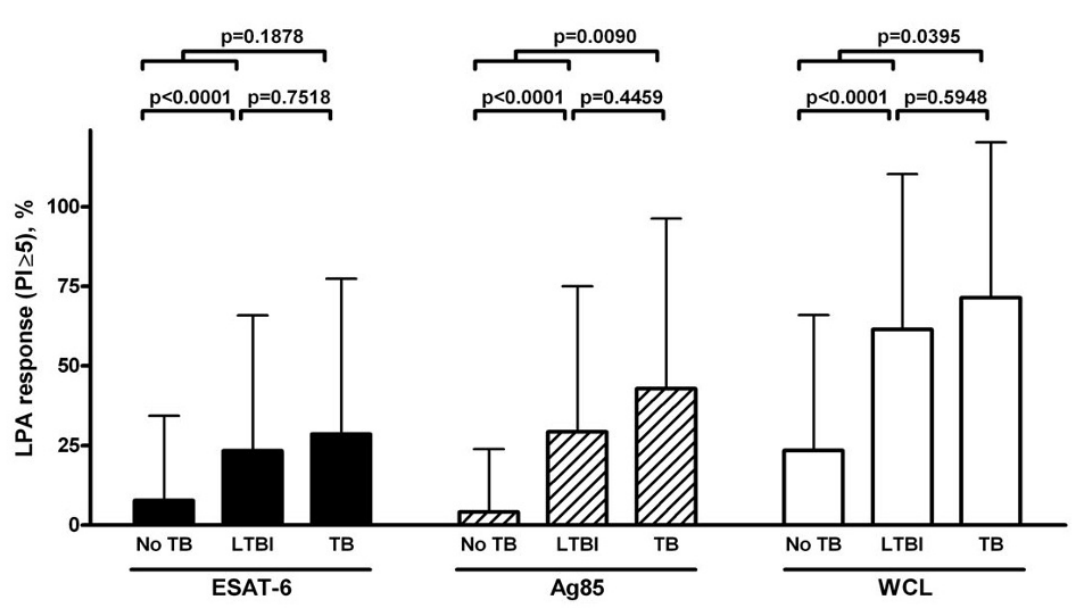

(B)

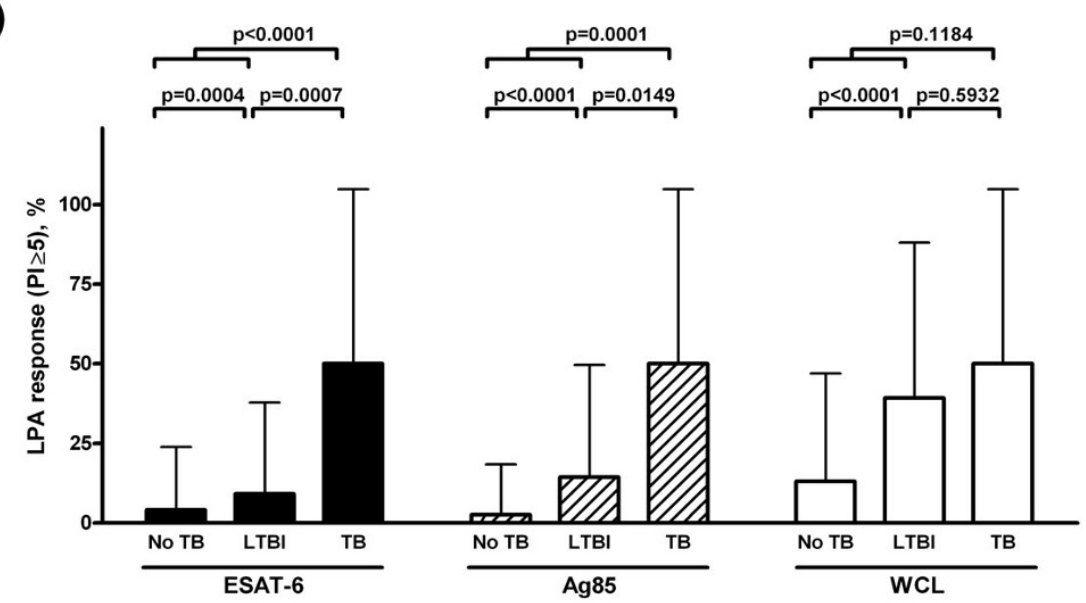

Figure I

Lymphocyte proliferation to mycobacterial antigens in subjects with a spectrum of tuberculosis. The percentage of subjects with lymphocyte proliferation index of $\geq 5$ to the indicated antigens is compared in subjects with active TB, latent TB and no TB in (A) all subjects, (B) subjects with a CD4 count $<350$ cells/ul, and (C) subjects with a CD4 count $\geq 350$ cells/ ul. The proliferation index is the ratio of counts per minute after stimulation with indicated antigen over counts per minute in unstimulated condition. (Ag85, antigen 85; ESAT-6, early secretory antigenic target 6; LTBI, latent tuberculosis infection; TB, tuberculosis; WCL, Mycobacterium tuberculosis whole cell lysate) 
Table 3: Prevalence of detectable lymphocyte proliferation to mycobacterial antigens in HIV-infected BCG-vaccinated adults with and without active TB.

\begin{tabular}{|c|c|c|c|c|}
\hline Antigen & $\begin{array}{l}\text { Subjects without TB } \\
\qquad(\mathrm{n}=13)\end{array}$ & $\begin{array}{l}\text { Subjects with latent TB } \\
\qquad(\mathrm{n}=635)\end{array}$ & $\begin{array}{l}\text { Subjects with active TB } \\
n=1206)\end{array}$ & P-value by ANOVA \\
\hline PHA, \% (n) & $543(45.0 \%)$ & $278(43.8 \%)$ & $8(61.5 \%)$ & 0.4175 \\
\hline MVS, \% (n) & $26(2.2 \%)$ & $21(3.3 \%)$ & $0(0 \%)$ & 0.2756 \\
\hline ESAT-6, \% (n) & $66(5.5 \%)$ & $84(13.2 \%)$ & $5(38.5 \%)$ & $<0.0001$ \\
\hline Ag85, \% (n) & $38(3.1 \%)$ & $119(18.7 \%)$ & $6(46.2 \%)$ & $<0.0001$ \\
\hline WCL, \% (n) & 207 (17.1\%) & $290(45.7 \%)$ & $8(61.5 \%)$ & $<0.0001$ \\
\hline
\end{tabular}

Ag85, antigen 85; ESAT-6, early secretory antigenic target 6; MVS, Mycobacterium vaccae sonicate; PHA, phytohemagglutinin; TB, tuberculosis; WCL, Mycobacterium tuberculosis whole cell lysate

vs. $26.6 \%, \mathrm{P}<0.0001 ;$ Ag85, 38.6\% vs. $24.8 \%, \mathrm{P}<0.0001$; WCL, $78.4 \%$ vs. $42.8 \%$, $\mathrm{P}<0.0001)$, but IFN- $\gamma$ responses were not consistently different between subjects with and without active TB (ESAT-6, 38.5\% vs. $26.6 \%, \mathrm{P}=0.3366$; Ag85, 23.1\% vs. 24.8\%, P = 0.8881; WCL, 76.9\% vs. $42.8 \%, \mathrm{P}=0.0133)$. There was, however, a clear differentiation between IFN- $\gamma$ responses to the positive control condition (PHA) compared to medium in all subjects (83.2\% vs. $18.6 \%, P<0.0001)$, in subjects without TB (85.0\% vs. $19.6 \%, \mathrm{P}<0.0001)$, in subjects with latent $\mathrm{TB}$ (79.7\% vs. $16.9 \%, \mathrm{P}<0.0001)$, and in subjects with active TB (69.2\% vs. $15.4 \%, P=0.0028)$. The absolute CD4 count correlated with the likelihood of having demonstrable IFN- $\gamma$ responses to each mycobacterial antigen: ESAT6, Pearson $\mathrm{r}=0.1076, \mathrm{P}<0.0001$; Ag85 Pearson $\mathrm{r}=$ $0.0488, \mathrm{P}<0.0356$; and WCL Pearson $\mathrm{r}=-0.0868, \mathrm{P}=$ 0.0002 . By contrast, the HIV viral load weakly impacted only the likelihood of positive responses to ESAT-6, Pearson $\mathrm{r}=0.0801, \mathrm{P}=0.0354$.

\section{Discussion}

An international effort is underway to find novel means of diagnosing and predicting TB in people with HIV [3]. We have shown that LPA responses to multiple mycobacterial antigens are enhanced during latent and active TB in HIVinfected subjects with CD4 counts $\geq 200$ cells $/ \mathrm{mm}^{3}$ and prior BCG immunization, and that these responses are less sensitive but more specific than TST in diagnosing active TB.

No previous studies have examined the diagnostic utility of LPA responses during active TB in HIV-infected adults. One group showed that LPA responses to mycobacteria were detectable in HIV-infected adults with TB, and noted a decline in response frequency at lower CD4 counts and in comparison to subjects without HIV [8]. However, only $67 \%$ of patients in that study were BCG immunized, LPA responses were not characterized according to BCG immunization or TST status, and the diagnostic operating characteristics of the test were not explored. In a prelimi-

Table 4: Operating characteristics of TST and LPA assays for the diagnosis of active TB.

\begin{tabular}{lccccccccccc}
\hline \multicolumn{1}{c}{} & \multicolumn{3}{c}{ All subjects } & \multicolumn{3}{c}{ Subjects with TST $5 \mathrm{~mm}$} \\
\hline & Sensitivity, \% & Specificity, \% & AUROC & PPV & NPV & Sensitivity, \% & Specificity, \% & AUROC & PPV & NPV \\
\hline TST & 84.6 & 65.5 & 0.7507 & 1.7 & 99.8 & -- & -- & -- & -- \\
\hline ESAT-6 & 38.5 & 92.0 & 0.6516 & 3.2 & 99.5 & 45.5 & 86.8 & 0.6611 & 5.6 & 98.9 \\
\hline Ag85 & 46.2 & 91.5 & 0.6882 & 3.68 & 99.6 & 54.6 & 81.3 & 0.6790 & 4.8 & 99 \\
\hline WCL & 61.5 & 73.0 & 0.6728 & 1.58 & 99.6 & 72.7 & 54.3 & 0.6353 & 2.68 & 99.1 \\
\hline
\end{tabular}

Ag85, antigen 85; AUROC, area under the receiver-operating characteristic curve, calculated as sensitivity plotted against I-specificity; ESAT-6, early secretory antigenic target 6; LPA, lymphocyte proliferation assay; NPV, negative predictive value; PPV, positive predictive value; TB, tuberculosis; TST, tuberculin skin test; WCL, Mycobacterium tuberculosis whole cell lysate 
Table 5: Odds of active TB according to lymphocyte proliferation assays, stratified by CD4 count and HIV viral load.

Odds of $\mathrm{TB}$ if $\mathrm{PI} \geq 5$ to Antigen

\begin{tabular}{lcccccccc}
\hline \multicolumn{3}{c}{ By CD4 count strata } & & \multicolumn{2}{c}{ By HIV Viral Load Quartile } \\
\hline Antigen & CD4 count $\geq 350^{*}$ & CD4 count $<350$ & $\begin{array}{c}\text { P value by Mantel-Haenszel } \\
\text { method }\end{array}$ & $\begin{array}{c}\text { Lowest } \\
\text { Second }\end{array}$ & Third & $\begin{array}{c}\text { Highest } \\
\text { P value by Mantel-Haenszel } \\
\text { method }\end{array}$ \\
\hline ESAT-6 & 15.8 & 2.9 & 0.0003 & 0 & 0 & 12.2 & 6.8 & 0.0030 \\
\hline Ag85 & 15.2 & 5.9 & $<0.0001$ & 0 & 0 & 11.2 & 7.1 & 0.0022 \\
\hline WCL & 3.3 & 4.8 & 0.0098 & 0 & 0 & 0 & 2.7 & 0.0333 \\
\hline
\end{tabular}

* Of subjects with active TB, six had CD4 counts $\geq 350$

Ag85, antigen 85; ESAT-6, early secretory antigenic target 6; PI, proliferative index; TB, tuberculosis; WCL, Mycobacterium tuberculosis whole cell lysate

nary report on seven of the subjects with HIV and active TB described in the present study we showed that LPA responses to mycobacterial antigens were detectable but did not determine the operating characteristics of the LPA nor the relation of these results to CD4 counts or HIV viral load [18]. Others have detailed LPA responses to PPD and

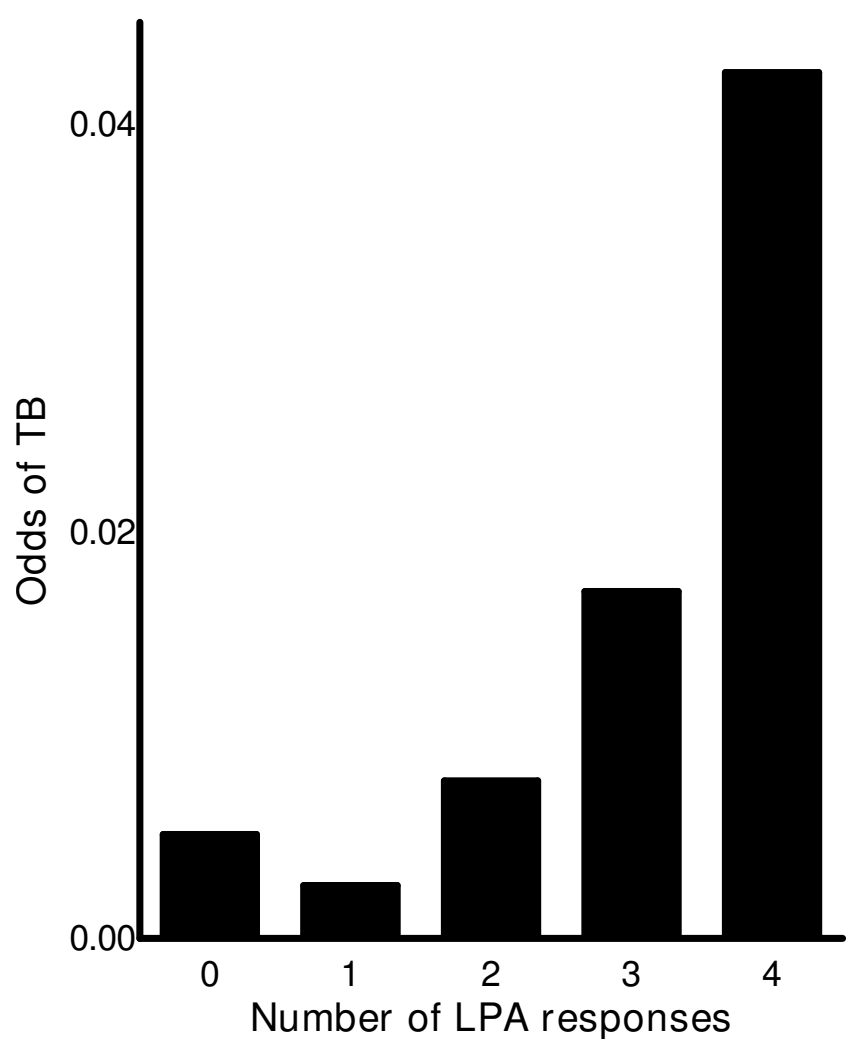

Figure 2

Odds of active TB according to number of detectable antimycobacterial lymphoproliferative responses.

(LPA, lymphocyte proliferation assays; TB, tuberculosis) mycobacterial cell cultures in HIV-infected adults with latent TB $[16,17]$, but these studies lack a description of the impact of HIV-related immunodeficiency or HIV viral load on assay results. Taken together, our data newly show that LPA responses to mycobacterial antigens are capable of detecting a spectrum of TB disease from latent to active and disseminated disease.

The ongoing search for novel TB diagnostic approaches in HIV-infected individuals results from the limitations of the TST in HIV-infected individuals, including decreased sensitivity at low CD 4 counts and the potential for false positive results after BCG vaccination [21-23]. Here, we have shown that LPA is less sensitive than TST, but more specific. Importantly, we did not examine the comparative sensitivity of LPA and TST at CD4 counts under 200 cells/ ul, since all subjects in this study had CD4 counts over 200 cells/ul. Since other immunological assays for the detection of TB have shown increased sensitivity when responses against multiple antigens were combined $[4,24]$, this approach has the potential to increase LPA sensitivity. The higher specificity of LPA likely results from the use of more TB-specific antigens ESAT-6 and Ag85, and is particularly important in BCG-immunized subjects such as are prevalent in areas of high TB endemicity. Even in subjects with a positive TST, subjects lacking LPA responses to mycobacterial antigens were highly unlikely to have active TB.

IGRA's are the current frontrunners in the race to replace TST $[4,5,25]$. The sensitivity of detectable LPA responses to mycobacterial antigens reported here is lower than that reported previously for IGRA assays during active TB. This may be due to different test characteristics of IGRA vs LPA, or the fact that commercial IGRA assays employ a mycobacterial antigen (CFP-10) that we did not use in the present LPA assay. IGRA sensitivity in HIV-infected subjects who recently completed therapy for TB was $71-81 \%$, 
whereas during culture positive TB in HIV-infected subjects the sensitivity of IGRA was $85 \%[26,27]$. In both studies, BCG vaccination was not universal, although in both papers the authors reported that BCG immunization had no impact on IGRA sensitivity.

Improving the sensitivity of LPA during active HIV-associated TB nonetheless will be critical, as the sensitivity values we observed are inadequate for clinical use. However, LPA sensitivity may be increased by using alternate TB antigens in combination, such as was done with IGRA [24]. Furthermore, we suspect the estimates of LPA sensitivity in our study are conservative, since the subjects with active $\mathrm{TB}$ reported here were diagnosed late after being scrutinized for clinical trial enrollment, and thus may have more clinically and immunologically subtle TB disease than would be identified during routine clinical care.

The specificity of LPA assays in the present study is similar to that of IGRA. IGRA specificity has been reported to be 98.6\% during active TB in HIV-negative subjects in Japan, [5], but in areas with high TB prevalence rates this figure is expected to be lower [28]. Accordingly, in BCG-immunized and HIV positive adults without active TB in Zambia, the specificity of IGRA was $71 \%$ for responses to ESAT- 6 alone and 57\% to ESAT- 6 and CPT- 10 combined [24]. These specificity values are lower than those we report for LPA, although no head-to-head comparison has yet been done. Since the TB epidemic flourishes largely in high prevalence areas where BCG vaccination is common, and in people with HIV co-infection, we believe it will be important to evaluate alternate platforms for diagnosing TB or TB risk in HIV-infected subjects from high TB prevalence areas [25].

Our data show that LPA responses are more predictive of TB risk in subjects with higher CD4 counts $[16,17]$. Importantly, all subjects in this study had CD4 counts above 200 cells $/ \mathrm{mm}^{3}$, so it will be important to evaluate the diagnostic utility of LPA in subjects with more profound HIV-related immunodeficiency. This is particularly important since IGRA based on the enzyme-linked immunospot (ELISPOT) technique appear not to lose sensitivity at lower CD4 counts $[29,30]$ although the impact of progressive HIV-related immunodeficiency on IGRA accuracy is still under study. While in prior studies antiretroviral therapy increased the likelihood of detectable LPA responses, patients in our cohort with higher HIV viral loads were more likely to have detectable lymphoproliferative responses to mycobacteria. Since antiretroviral therapy was rare in our cohort, the viral loads reported here reflect untreated values, and thus we posit that the relation between the HIV viral load and TB risk is different in untreated vs. treated subjects. It is possible that a high HIV viral load off therapy may contribute to greater HIV- related immunological dysfunction and thus vulnerability to TB disease, whereas treated patients with a lower viral load on therapy may be more likely to have detectable responses via antimycobacterial immune reconstitution. It is also possible that the higher viral loads were a consequence of new active TB [31].

HIV and TB intersect most lethally in the developing world. Thus, it is important to consider whether conducting the LPA - a complicated five day assay involving lowlevel radioactivity - is feasible in the developing world. We generated these data in Tanzania, but the assessment of lymphocyte radioactivity (which is proportionate to proliferation in this assay) was carried out in Finland. Further, all assays were conducted through a US-funded clinical trial with an intensive focus on quality control. Accordingly, we suspect that only larger medical centers in the developing world would have the capacity to conduct LPA assays.

Despite an extensive literature that suggests that IFN- $\gamma$ responses to mycobacterial antigens are decreased but detectable in HIV-infected individuals $[8,10,14,26,27]$, we did not see differences in IFN- $\gamma$ responses in subjects with and without TB. One reason for this discrepancy may be differential assay duration. While the IFN- $\gamma$ results here stem from five day incubation assays, currently commercially available assays employ one day of incubation. One day assays measure effector cell IFN- $\gamma$ release, while five day assays likely measure IFN- $\gamma$ release from effector and central memory $\mathrm{T}$ cells $[32,33]$. As a result, our five day IFN- $\gamma$ assays may fail to discriminate between subjects with novel effector $\mathrm{T}$ cell responses generated during active $\mathrm{TB}$ and those with pre-existing memory $\mathrm{T}$ cell responses to TB. Despite careful attention to quality control in this developing world setting, a small proportion of IFN- $\gamma$ assays lacked PHA responses or had detectable responses to the medium control. Assay failure thus has the potential to contribute to our IFN- $\gamma$ assay's inability to detect differences between subjects with active TB or no $\mathrm{TB}$, although notably the assay did discriminate between subjects with latent TB and no TB. Another potential reason for the discrepant IFN- $\gamma$ results in this study is that certain mycobacterial antigens may be more efficient at generating IFN- $\gamma$ responses than others. Commercially available assays of IFN- $\gamma$ target ESAT- 6 as well as CFP-10 with and without TB7.7 $[4,25]$, and others have demonstrated detectable IFN- $\gamma$ responses in HIV-infected subjects with active $\mathrm{TB}$ using purified protein derivative (PPD) $[8,14]$ Our assay, which assessed responses to ESAT-6, Ag85 and WCL, may be less likely to generate IFN- $\gamma$ responses that distinguish between subjects with and without TB. At a minimum, our data suggest that incubation period and antigen choice are critical variables in IFN- $\gamma$ assays for TB. 
Our data show that detectable LPA responses to TB are associated with TB infection, but not necessarily with effective cellular immune protection against disease. This is consistent with the known association between TST results and TB risk [34,35]. This model has important implications regarding the conduct of TB vaccine trials: it will be vitally important to determine not only whether $\mathrm{TB}$ vaccines generate antimycobacterial immune responses, but whether these immune responses correlate with disease protection.

\section{Conclusion}

In conclusion, an assay of antimycobacterial lymphocyte proliferation was more specific but less sensitive than TST in detecting active TB in HIV-infected and BCG-immunized patients from a high TB prevalence area. Given the potential for improving LPA sensitivity by using a different panel of mycobacterial antigens and assessing it in subjects with more clinically overt active TB, LPA merits continued evaluation as a novel diagnostic tool for HIVassociated TB.

\section{Competing interests}

The authors declare that they have no competing interests.

\section{Authors' contributions}

TL helped design the study, performed data analysis and drafted the manuscript; MM helped with data acquisition and manuscript preparation; LM helped with data acquisition and manuscript preparation; MB helped with data acquisition and manuscript preparation; KP helped with data acquisition and manuscript preparation; CFR conceived the study, participated in its design and conduct, and helped to draft the manuscript. All authors read and approved the final manuscript.

\section{About the authors}

The authors of this manuscript participate in the Dar Dar Health Study, a randomized placebo controlled trial of whole inactivated Mycobacterium vaccae, a novel TB booster vaccine given to 2,000 HIV-infected adults in Dar es Salaam, Tanzania.

\section{Acknowledgements}

Presented at: 15th Conference on Retroviruses and Opportunistic Infections, Boston, MA, February 2008

This study was supported by the National Institutes of Health, DAIDS, AI 45407, and the Fogarty International Center, D43-TW006807. Beyond their initial decision to support the study, these organizations did not control study design; the collection, analysis and interpretation of the data; the writing of the manuscript; or the decision to submit the manuscript.

We thank Jenni Vuola for immunological assay development and longstanding fruitful collaboration.

\section{References}

I. Dye C: Global epidemiology of tuberculosis. Lancet 2006, 367(95 I 4):938-940.

2. Dowdy DW, Chaisson RE, Moulton LH, Dorman SE: The potential impact of enhanced diagnostic techniques for tuberculosis driven by HIV: a mathematical model. Aids 2006, 20(5):75I-762.

3. The global plan to stop TB, 2006-2015/Stop TB Partnership [http://www.stoptb.org/globalplan/assets/documents/GlobalPlanFi nal.pdf]

4. Menzies D, Pai M, Comstock G: Meta-analysis: new tests for the diagnosis of latent tuberculosis infection: areas of uncertainty and recommendations for research. Ann Intern Med 2007, I 46(5):340-354.

5. Mori T, Sakatani M, Yamagishi F, Takashima T, Kawabe Y, Nagao K, Shigeto E, Harada N, Mitarai S, Okada M, et al.: Specific detection of tuberculosis infection: an interferon-gamma-based assay using new antigens. Am J Respir Crit Care Med 2004, I 70(I):59-64.

6. Doherty TM, Demissie A, Olobo J, Wolday D, Britton S, Eguale T, Ravn P, Andersen P: Immune responses to the Mycobacterium tuberculosis-specific antigen ESAT-6 signal subclinical infection among contacts of tuberculosis patients. J Clin Microbiol 2002, 40(2):704-706.

7. Diel R, Loddenkemper R, Meywald-Walter K, Niemann S, Nienhaus A: Predictive value of a whole blood IFN-gamma assay for the development of active tuberculosis disease after recent infection with Mycobacterium tuberculosis. Am J Respir Crit Care Med 2008, I77( 1 0): I I64-I I 70.

8. Silveira H, Ordway D, Dockrell H, Jackson M, Ventura F: Cell-mediated immune responses to mycobacterial antigens in patients with pulmonary tuberculosis and HIV infection. Clin Exp Immunol I 997, I I O(I):26-34.

9. Sterling TR, Dorman SE, Chaisson RE, Ding L, Hackman J, Moore K, Holland SM: Human immunodeficiency virus-seronegative adults with extrapulmonary tuberculosis have abnormal innate immune responses. Clin Infect Dis 200I, 33(7):976-982.

10. Rangaka MX, Diwakar L, Seldon R, van Cutsem G, Meintjes GA, Morroni C, Mouton P, Shey MS, Maartens G, Wilkinson KA, et al.: Clinical, immunological, and epidemiological importance of antituberculosis $\mathrm{T}$ cell responses in HIV-infected Africans. Clin Infect Dis 2007, 44(I 2): 1639-1646.

II. McDyer JF, Hackley MN, Walsh TE, Cook JL, Seder RA: Patients with multidrug-resistant tuberculosis with low CD4+ $T$ cell counts have impaired ThI responses. I Immunol 1997, I 58(I):492-500.

12. Zhang M, Gong J, lyer DV, Jones BE, Modlin RL, Barnes PF: T cell cytokine responses in persons with tuberculosis and human immunodeficiency virus infection. I Clin Invest 1994, 94(6):2435-2442

13. Winkler S, Necek M, Winkler H, Adegnika AA, Perkmann T, Ramharter M, Kremsner PG: Increased specific $T$ cell cytokine responses in patients with active pulmonary tuberculosis from Central Africa. Microbes Infect 2005, 7(9-1 0): I I6I-I I69.

14. Oh MD, Kang Cl, Kim US, Kim NJ, Lee B, Kim HB, Choe KW: Cytokine responses induced by Mycobacterium tuberculosis in patients with HIV-I infection and tuberculosis. Int $J$ Infect Dis 2005, 9(2): II0-II6.

15. Wood R, Maartens G, Lombard CJ: Risk factors for developing tuberculosis in HIV-I-infected adults from communities with a low or very high incidence of tuberculosis. I Acquir Immune Defic Syndr 2000, 23(I):75-80.

16. Schluger NW, Perez D, Liu YM: Reconstitution of immune responses to tuberculosis in patients with HIV infection who receive antiretroviral therapy. Chest 2002, I 22(2):597-602.

17. Sutherland R, Yang H, Scriba T], Ondondo B, Robinson N, Conlon C, Suttill A, McShane H, Fidler S, McMichael A, et al:: Impaired IFNgamma-secreting capacity in mycobacterial antigen-specific CD4 T cells during chronic HIV-I infection despite longterm HAART. Aids 2006, 20(6):82I-829.

18. Mtei L, Matee M, Herfort O, Bakari M, Horsburgh CR, Waddell R, Cole BF, Vuola JM, Tvaroha S, Kreiswirth B, et al.: High rates of clinical and subclinical tuberculosis among HIV-infected ambulatory subjects in Tanzania. Clin Infect Dis 2005, 40(10): $1500-1507$. 
19. Matee M, Lahey T, Vuola JM, Mtei L, Cole BF, Bakari M, Arbeit RD, Horsburgh CR, Pallangyo K, von Reyn CF: Baseline mycobacterial immune responses in HIV-infected adults primed with bacille Calmette-Guerin during childhood and entering a tuberculosis booster vaccine trial. J Infect Dis 2007, 195(I):I18-123.

20. Vuola JM, Ristola MA, Cole B, Jarviluoma A, Tvaroha S, Ronkko T, Rautio O, Arbeit RD, Reyn CF: Immunogenicity of an inactivated mycobacterial vaccine for the prevention of HIV-associated tuberculosis: a randomized, controlled trial. Aids 2003, I7(16):235I-2355.

21. Joos TJ, Miller WC, Murdoch DM: Tuberculin reactivity in bacille Calmette-Guerin vaccinated populations: a compilation of international data. Int J Tuberc Lung Dis 2006, I0(8):883-89।.

22. Markowitz N, Hansen NI, Wilcosky TC, Hopewell PC, Glassroth J, Kvale PA, Mangura BT, Osmond D, Wallace JM, Rosen MJ, et al.: Tuberculin and anergy testing in HIV-seropositive and HIVseronegative persons. Pulmonary Complications of HIV Infection Study Group. Ann Intern Med 1993, I I 9(3): 185-193.

23. Menzies R, Vissandjee B: Effect of bacille Calmette-Guerin vaccination on tuberculin reactivity. Am Rev Respir Dis 1992 145(3):62I-625.

24. Chapman AL, Munkanta M, Wilkinson KA, Pathan AA, Ewer K, Ayles $\mathrm{H}$, Reece $\mathrm{WH}$, Mwinga A, Godfrey-Faussett P, Lalvani A: Rapid detection of active and latent tuberculosis infection in HIV. positive individuals by enumeration of Mycobacterium tuberculosis-specific T cells. Aids 2002, 16(17):2285-2293

25. Pai M, Dheda K, Cunningham J, Scano F, O'Brien R: T-cell assays for the diagnosis of latent tuberculosis infection: moving the research agenda forward. Lancet Infect Dis 2007, 7(6):428-438.

26. Bellete B, Coberly J, Barnes GL, Ko C, Chaisson RE, Comstock GW, Bishai WR: Evaluation of a whole-blood interferon-gamma release assay for the detection of Mycobacterium tuberculosis infection in 2 study populations. Clin Infect Dis 2002, 34(II): | 1449-I 456.

27. Tsiouris SJ, Coetzee D, Toro PL, Austin J, Stein Z, El-Sadr W: Sensitivity analysis and potential uses of a novel gamma interferon release assay for diagnosis of tuberculosis. J Clin Microbiol 2006, 44(8):2844-2850.

28. Kang YA, Lee HW, Yoon HI, Cho B, Han SK, Shim YS, Yim J]: Discrepancy between the tuberculin skin test and the wholeblood interferon gamma assay for the diagnosis of latent tuberculosis infection in an intermediate tuberculosis-burden country. Jama 2005, 293(22):2756-276I.

29. Lawn SD, Bangani N, Vogt M, Bekker LG, Badri M, Ntobongwana M Dockrell HM, Wilkinson RJ, Wood R: Utility of interferongamma ELISPOT assay responses in highly tuberculosisexposed patients with advanced HIV infection in South Africa. BMC Infect Dis 2007, 7:99.

30. Clark SA, Martin SL, Pozniak A, Steel A, Ward B, Dunning J, Henderson DC, Nelson M, Gazzard B, Kelleher P: Tuberculosis antigenspecific immune responses can be detected using enzymelinked immunospot technology in human immunodeficiency virus (HIV)-I patients with advanced disease. Clin Exp Immunol 2007, I 50(2):238-244.

31. Toossi Z, Mayanja-Kizza $\mathrm{H}$, Hirsch CS, Edmonds KL, Spahlinger T, Hom DL, Aung H, Mugyenyi P, Ellner JJ, Whalen CW: Impact of tuberculosis (TB) on HIV-I activity in dually infected patients. Clin Exp Immunol 200I, I 23(2):233-238.

32. Lalvani $A$ : Counting antigen-specific $T$ cells: a new approach for monitoring response to tuberculosis treatment? Clin Infect Dis 2004, 38(5):757-759.

33. Carrara S, Vincenti D, Petrosillo N, Amicosante M, Girardi E, Goletti $D$ : Use of a T cell-based assay for monitoring efficacy of antituberculosis therapy. Clin Infect Dis 2004, 38(5):754-756.

34. Guelar A, Gatell JM, Verdejo J, Podzamczer D, Lozano L, Aznar E, Miro JM, Mallolas J, Zamora L, Gonzalez J, et al: A prospective study of the risk of tuberculosis among HIV-infected patients. Aids 1993, 7(10): 1345-1349.

35. Colebunders RL, Lebughe I, Nzila N, Kalunga D, Francis H, Ryder R, Piot P: Cutaneous delayed-type hypersensitivity in patients with human immunodeficiency virus infection in Zaire. J Acquir Immune Defic Syndr 1989, 2(6):576-578.

\section{Pre-publication history}

The pre-publication history for this paper can be accessed here:

http://www.biomedcentral.com/1471-2334/9/21/prepub
Publish with Biomed Central and every scientist can read your work free of charge

"BioMed Central will be the most significant development for disseminating the results of biomedical research in our lifetime. "

Sir Paul Nurse, Cancer Research UK

Your research papers will be:

- available free of charge to the entire biomedical community

- peer reviewed and published immediately upon acceptance

- cited in PubMed and archived on PubMed Central

- yours - you keep the copyright
BioMedcentral 